\title{
Cohesión familiar y factores relacionados en adolescentes escolarizados
}

\author{
Family cohesion and related factors in school adolescents \\ Santiago Gómez-Velásquez ${ }^{*}$ orcid.org/0000-0002-5618-2056 \\ Giselly Matagira-Rondón² orcid.org/0000-0001-9642-1253 \\ Maite Catalina Agudelo-Cifuentes² orcid.org/0000-0003-1501-9452 \\ Dedsy Yajaira Berbesi-Fernández² orcid.org/0000-0002-1716-957X \\ Santiago Alberto Morales-Mesa² orcid.org/0000-0002-8987-7399
}

1. Facultad de Nutrición, Universidad CES. Medellín, Colombia.

2. Facultad de Enfermería, Universidad CES. Medellín, Colombia.

\section{Resumen}

Introducción: El nivel de cohesión de un núcleo familiar permite caracterizar los vínculos y relaciones que existen entre sus miembros y constituye un insumo fundamental para el desarrollo de políticas y programas de intervención en la familia. Objetivo: Identificar factores asociados a la cohesión familiar en adolescentes del Municipio de Envigado. Materiales y métodos: Estudio transversal, de fuente de información primaria, con una muestra de 1008 estudiantes de dos colegios de Envigado. Se utilizó la escala de cohesión familiar, de Depresión de Zung, de Habilidades Sociales de Gismero, y preguntas sociodemográficas y de consumo de sustancias. Resultados: El 61,5\% de los adolescentes se encontraban sin riesgo de alcoholismo, el 73,8\% sin dependencia a drogas, un 16,5\% tenían riesgo de depresión y un 81,7\% presentaban buenas habilidades sociales. La prevalencia de cohesión familiar fue 96,6\%. La satisfacción con la familia y la ausencia de depresión, se asociaron a buena cohesión familiar. Conclusiones: La presencia de depresión en el adolescente y un alto nivel de insatisfacción con el núcleo familiar son factores asociados a la falta de cohesión familiar; factores como la violencia intrafamiliar, las habilidades sociales y el consumo de sustancias psicoactivas no presentaron asociación con la variable de interés.

Palabras clave: Adolescencia; cohesión familiar; satisfacción familiar; depresión. (Fuente: DeCS, Bireme).

\begin{abstract}
Introduction: The level of cohesion within a family nucleus is useful to characterize connections and relationships established between its members. It also constitutes a fundamental factor in the development of family intervention programs and policies. Objective: To identify factors associated with family cohesion in adolescents from the municipality of Envigado (Colombia). Materials and methods: A cross-sectional study using a primary source of information and a sample of 1008 students from two schools from Envigado. The following instruments were used: family cohesion scale; Zung's Depression scale; Gismero's Social Skills scale; and sociodemographic and substance use questions. Results: $61.5 \%$ of adolescents were not at risk for alcoholism, $73.8 \%$ were not drug dependent, $16.5 \%$ were at risk of depression, and $81.7 \%$ had adequate social skills. The prevalence of family cohesion was $96.6 \%$. A feeling of satisfaction with their families and absence of depression were associated with good family cohesion. Conclusions: Depression in adolescents and a high level of dissatisfaction towards their family are factors associated with the lack of family cohesion. Factors such as domestic violence, social skills, and use of psychoactive substances did not show an association with the variable of interest.
\end{abstract}

Keywords: Adolescence; family cohesion; family satisfaction; depression. (Source: DeCS, Bireme). 


\section{Introducción}

La adolescencia es un período de maduración biológica, psicológica y social necesaria para la adaptación a la edad adulta; este proceso decisivo no se da igual para todos los adolescentes y dependerá no sólo de circunstancias biológicas sino también de aquellas de tipo social, personal y cultural(1).

El desarrollo integral del adolescente se relaciona significativamente con factores que son externos a su individualidad, en lo particular, el contexto familiar es una dimensión de interés central que puede aportar elementos para el análisis de la adolescencia como parte del ciclo vital. Desde el ejercicio científico, los estudios han evidenciado como los jóvenes construyen, modifican o resignifican sus representaciones e imaginarios en función con su relación en el hogar(2).

Actualmente, hay diferentes formas en la composición familiar (nucleares, monoparentales, extensas y anucleares), en la relación que se establecen entre los diferentes miembros de la familia y en la forma que encuentran para vincularse(3). En este aspecto, la cohesión familiar es un indicador de satisfacción e identidad, que desarrollan los miembros de una familia con respecto al lazo sentimental y emocional que los une(4); este vínculo no sólo potencializa la unión familiar sino que es también transcendental para el desarrollo psicosocial de todos los miembros de la familia y en los adolescentes, es un factor predictivo de salud mental(5).

La cohesión familiar se afecta cuando no hay cercanía o vínculo entre los miembros de la familia, ya sea por relaciones distantes o consecuencia del fraccionamiento por la violencia intrafamiliar que puede estar mediada o intensificada por el uso de drogas, la satisfacción que exista entre los miembros de la familia, o las habilidades sociales que tienen los miembros del hogar frente a circunstancias retadoras o estresantes(6,7).

El nivel de cohesión de un núcleo familiar permite caracterizar los vínculos y relaciones que concurren entre sus miembros, en donde la identificación de los factores que se asocian con la cohesión familiar, puede ser un insumo fundamental para el desarrollo de políticas y programas de intervención, que, desde un nivel de atención primaria, se orienten a intervenir positivamente las condiciones de salud de una comunidad, trabajando la familia como parte constitutiva de la estructura social(8).

En cuanto a la depresión en el adolescente, es posible plantearlo como un factor que se ha mostrado relacionado con elementos generados de forma directa o indirecta por entornos familiares carentes de cohesión(9); de igual manera, el consumo de sustancias sicoactivas por parte del adolescente ha demostrado generar impactos negativos en las dinámicas familiares(10), mientras que investigaciones recientes muestran una correlación positiva entre habilidades sociales y funcionalidad familiar(11).

Con base en lo anterior, identificar factores en la población adolescente que se relacionen con las dinámicas de cohesión familiar aporta una información importante para entender los diferentes contextos en que puede transitar el desarrollo del adolescente; en la ciudad de Medellín se han realizado estudios con adolescentes escolarizados, donde se ha identificado que la cohesión familiar es una característica que se asocia de manera significativa a distintos aspectos de su salud mental(12) como la depresión(13) y el consumo de sustancias psicoactivas(12). Por lo anteriormente expuesto, el objetivo de este trabajo es identificar los factores asociados a la cohesión familiar en adolescentes del Municipio de Envigado, Colombia para el año 2014, que permita generar información que aporte a la planeación de programas dirigidos a mejorar las relaciones disfuncionales entre padres e hijos y la estructuración de los lazos familiares.

\section{Materiales y métodos}

Se realizó un estudio de enfoque empírico analítico, de diseño transversal, con metodología cuantitativa, utilizando fuente de información primaria. La muestra fue calculada utilizando EPIDAT 4.2, con una proporción esperada de $50 \%$, nivel de confianza de 95\% y un límite de confianza de 3\%, obteniéndose un tamaño de muestra de 1008 personas, de los cuales 508 estudiantes fueron seleccionados de colegios oficiales y 500 de colegios privados en el municipio de Envigado; teniendo como criterio de inclusión adolescentes con edades comprendidas entre 12 y 18 años, con lugar de residencia dicho municipio. Se excluyeron los adolescentes que al momento de la encuesta estuvieran bajo el efecto de sustancias psicoactivas (identificados por observación directa del encuestador, o por información del profesor 
encargado); también fueron excluidos los adolescentes con limitaciones mentales manifiestas y evidentes.

Se realizó muestreo probabilístico, con estratificación según la naturaleza de la institución (público y privado), la selección fue bietápica, por medio de selección aleatoria simple, en la primera etapa se seleccionaron las instituciones y en la segunda los grados escolares a encuestar.

Se utilizó como técnica de recolección la encuesta, la cual fue diseñada utilizando la herramienta Google Forms, para el acceso a la encuesta se utilizaron las salas de cómputo de cada institución educativa y durante cada sesión se contó con el acompañamiento de un profesional en psicología y la supervisión de los investigadores quienes orientaron permanentemente el diligenciamiento; dentro de la encuesta se incluyeron preguntas demográficas, personales, familiares y de consumo de sustancias. Para medir la cohesión familiar se utilizó la escala de cohesión familiar validada para Colombia por integrantes del Grupo de Investigación en Salud Mental, la cual mostró buena consistencia interna (Alpha de Cronbach de 0,798)(14). Adicionalmente, se indagó por aspectos relacionados a violencia intrafamiliar. Para evaluar consumo de sustancias, se utilizó la escala de DUSI (por las siglas en inglés: Drug Use Screening Inventory) Alcohol y DUSI Drogas (Marihuana), la versión modificada por Yolanda Torres de Galvis(15), que cuenta con 10 dominios relacionados con el riesgo para uso de alcohol y otras drogas. Para evaluar depresión se utilizó la escala Zhung, la cual cuantifica síntomas depresivos; dicha escala fue validada para su uso en adolescentes escolarizados por el grupo de salud mental de la Universidad de Cartagena (Alpha de Cronbach de 0,689). Las habilidades sociales fueron medidas por medio de la Escala de Habilidades sociales de Elena Gismero(16), que cuenta con 33 ítem, 28 de los cuales están redactados en el sentido de falta de aserción o déficit de habilidades sociales y 5 de ellos en sentido positivo, con 4 opciones de respuesta; este instrumento fue validado para ser usado en adolescentes $^{(17) .}$

Para el procesamiento de la información se utilizó el programa estadístico para ciencias de la salud SPSS versión 21 (Licencia Universidad CES). Se realizó análisis univariado con el fin de establecer las características demográficas, psicológicas y familiares de los adolescentes; análisis bivariado para determinar las características asociadas a la cohesión familiar, por medio de regresión logística, obteniendo los Odds Ratio (OR) con sus respectivos intervalos de confianza del $95 \%$ y valores $p<0,05$; para el modelo ajustado se ingresaron las variables que cumplieron el criterio de Hosmer-Lemeshow con valores de $\mathrm{p}<0,25$.

\section{Consideraciones éticas}

Esta investigación fue aprobada por el Comité de Ética de la Universidad CES, en sesión 73 del 25 de agosto del 2014. Se siguieron los requerimientos de las Normas Científicas, Técnicas y Administrativas para la Investigación en Salud, los participantes firmaron consentimiento informado; según la Resolución 008430 del 4 de octubre de 1993 del Ministerio de Salud de Colombia, clasificada como investigación con riesgo mínimo.

\section{Resultados}

En cuanto a las características sociodemográficas de los adolescentes participantes del estudio, la mayoría se encontraban entre 12 y 15 años (59\%), el $41 \%$ restante tenían entre 16 y 18 años. Así mismo, se contó con una mayor participación de hombres $(60 \%)$ que de mujeres.

Respecto a la institución educativa, se observó que el $61,4 \%$ de los estudiantes encuestados pertenecían a colegios públicos y el $38,6 \%$ a colegios privados. Además, de acuerdo con la ubicación de la institución, la mayoría de los participantes se encontraban en colegios ubicados en el área urbana (91\%) y sólo un $9 \%$ en área rural.

De otro lado, respecto al nivel educativo en el que se encontraban los estudiantes participantes de la investigación, el $41,7 \%$ se encontraban cursando octavo o noveno, el $35,5 \%$ decimo o undécimo y el $22,8 \%$ sexto o séptimo.

De acuerdo con las características personales de los adolescentes, se identificó que un 16,5\% de estos tenían riesgo de depresión, y el 83,5\% restante se encontraban en un estado óptimo respecto a depresión. En este sentido, por medio de la escala Zhung se observó que un 23,8\% de los participantes manifestó sentirse triste muchas veces o siempre, y el $52,2 \%$ a veces. Así mismo, un 24,5\% manifestó sentir ganas de llorar y llorar muchas veces o siempre, y un $40,3 \%$ a veces. También llamó la atención, que un $8,2 \%$ de los adolescentes expresó que, en el último 
año, nunca sintió ganas ni deseo de compartir con otras personas. Un $15,1 \%$ de los adolescentes manifestó que muchas veces o siempre se cansa sin motivos y un $18,3 \%$ indicó que su mente nunca se encuentra despejada. Se resalta que un $27,1 \%$ de los adolescentes manifestó siempre o muchas veces sentirse inquieto $y$ no poder mantener la tranquilidad, así mismo un $13,6 \%$ nunca sentir confianza en el futuro, un 7,4\% nunca encontrar agradable vivir y un $5,9 \%$ sentir siempre que sería mejor para los demás si estuviera muerto.

En cuanto a las habilidades sociales medidas por medio de la Escala de Habilidades sociales de Elena Gismero, se encontró que el $81,7 \%$ de los adolescentes encuestados tenía buenas habilidades sociales. Al respecto, específicamente se identificó que el 63,6\% de los adolescentes no evita asistir a reuniones sociales por miedo a hacer o decir tonterías, a un $39 \%$ no le cuesta expresar sus sentimientos a los demás y la mitad manifestó que no tiene dificultad para expresar sus opiniones en grupos como clases, reuniones y demás. Adicionalmente, se observó que al 44,4\% no le cuesta expresar enfado hacia el otro sexo cuando tiene motivos y a un $8 \%$ no le cuesta hacerlo ante un familiar. Asimismo, un 42,6\% no tienen dificultad en expresarle a alguien que no le apetece volver a salir con esa persona.

En cuanto al consumo, se observó que el $9 \%$ de los adolescentes participantes del estudio tenían alcoholismo y un 29,5\% riesgo de alcoholismo, entre tanto, un poco más de la mitad de estos, el $61,5 \%$ no tenían problemas con el alcohol. Específicamente, por medio de la escala DUSI Alcohol, se observó que, en el último año, el 81,6\% de los adolescentes no había sentido en ningún momento deseo intenso de consumir alcohol; además, un 93,8\% de los participantes indicaron que en ningún momento habían sentido que no pudieran controlar el consumo de alcohol. Adicionalmente, se encontró que el 88,9\% de los adolescentes no se habían saltado ninguna regla por estar bajo el efecto del consumo de alcohol y casi la totalidad $(98,6 \%)$ manifestó que no habían sufrido accidentes de tránsito por estar bajo consumo de alcohol.

De otro lado, en cuanto al consumo de drogas, el 5,1\% fueron clasificados con dependencia a drogas y el $21,1 \%$ con alto riesgo de dependencia a drogas, mientras que el 73,8\% no tenían problemas frente al consumo. A través de la escala DUSI drogas, se identificó que la mayoría de los participantes en el último año no había sentido en ningún momento que no pudiera controlar el consumo de drogas $(95,5 \%)$, además, el $94,1 \%$ no había pasado por alto reglas por estar bajo el consumo drogas y el 98,2\% no había sufrido accidentes por esta causa.

Respecto a lo familiar, se identificó que un 50,8\% de los adolescentes manifestaron percibir violencia intrafamiliar en sus hogares; a pesar de esto, un $85,8 \%$ se percibían como satisfechos con su familia.

\section{Prevalencia de cohesión familiar}

Se identificó una prevalencia de cohesión familiar del $96,6 \%$. De los adolescentes que mostraron buena cohesión familiar, el 59,2\% tenían entre 12 y 15 años, sin ser significativa la diferencia con los de 16 a 18 años $(p=0,630)$. El $60,6 \%$ de los adolescentes con buena cohesión familiar eran hombres, encontrándose significativa la diferencia respecto a las mujeres $(p=0,037)$. Asimismo, se observó diferencia significativa respecto a la prevalencia de cohesión familiar en los adolescentes de colegios privados y públicos, siendo mayor la cohesión en los públicos $(p=0,009)$. En los adolescentes que mostraron buena cohesión familiar, fue más frecuente que el colegio estuviera en zona urbana $(90,8 \%)$ y que se encontraran en octavo y noveno grado (42,4\%); sin embargo, ambas características no se asocian significativamente a la cohesión familiar $(p>0,005)$ (Tabla 1$)$.

Se observó que el $88 \%$ de los adolescentes de familias con adecuada cohesión se sentían satisfechos con la familia, mientras que en quienes convivían con familias con baja cohesión, se percibieron principalmente insatisfechos, siendo estas diferencias estadísticamente significativas $(p<0,001)$. De igual forma, la cohesión familiar se mostró estrechamente asociada con la presencia o ausencia de violencia familiar $(p=0,005)$, con el déficit de habilidades sociales $(p=0,003)$ siendo mayor el porcentaje de ausencia de déficit en adolescentes provenientes de familias con adecuada cohesión. En los adolescentes convivientes con familias de baja cohesión, fue mucho mayor el porcentaje de presencia de riesgo de depresión, alcoholismo y dependencia de drogas $(p<0,001)$ (Tabla 1$)$. 
Tabla 1. Características asociadas a la cohesión familiar en adolescentes escolarizados de envigado, 2014

\begin{tabular}{|c|c|c|c|c|c|c|}
\hline \multirow{2}{*}{\multicolumn{2}{|c|}{ Característica }} & \multicolumn{4}{|c|}{ Cohesión familiar } & \multirow{2}{*}{ Valor $p^{*}$} \\
\hline & & \multirow{2}{*}{$\begin{array}{l}\text { Si } \\
529\end{array}$} & \multirow{2}{*}{$\begin{array}{l}\% \\
59,2\end{array}$} & \multirow{2}{*}{$\begin{array}{l}\text { No } \\
17\end{array}$} & \multirow{2}{*}{$\begin{array}{r}\% \\
54,8\end{array}$} & \\
\hline Edad & 12-15 años & & & & & 0,630 \\
\hline & 16-18 años & 365 & 40,8 & 14 & 45,2 & \\
\hline \multirow[t]{2}{*}{ Sexo } & Masculino & 542 & 60,6 & 13 & 41,9 & 0,037 \\
\hline & Femenino & 352 & 39,4 & 18 & 58,1 & \\
\hline \multirow[t]{2}{*}{ Tipo de colegio } & Público & 542 & 60,6 & 26 & 84 & 0,009 \\
\hline & Privado & 352 & 39,4 & 5 & 16,1 & \\
\hline \multirow[t]{2}{*}{ Zona colegio } & Urbana & 812 & 90,8 & 30 & 96,8 & 0,515 \\
\hline & Rural & 82 & 9,2 & 1 & 3,2 & \\
\hline \multirow[t]{3}{*}{ Grado de escolaridad } & Sexto-Séptimo & 201 & 22,5 & 10 & 32 & 0,085 \\
\hline & Octavo-Noveno & 379 & 42,4 & 7 & 22,6 & \\
\hline & Décimo-Undécimo & 314 & 35,1 & 14 & 45,2 & \\
\hline \multirow[t]{2}{*}{ Satisfacción con la familia } & Satisfecho & 787 & 88 & 7 & 22.6 & 0,000 \\
\hline & Insatisfecho & 107 & 12 & 24 & 77,4 & \\
\hline \multirow[t]{2}{*}{ Violencia intrafamiliar } & Ausencia & 462 & 51,7 & 8 & 25,8 & 0,005 \\
\hline & Presencia & 432 & 48,3 & 23 & 74,2 & \\
\hline \multirow[t]{2}{*}{ Déficit habilidades sociales } & No & 737 & 82,4 & 19 & 61,3 & 0,003 \\
\hline & $\mathrm{Si}$ & 157 & 17,6 & 12 & 38,7 & \\
\hline \multirow[t]{2}{*}{ Depresión } & No & 761 & 85,1 & 11 & 35,5 & 0,000 \\
\hline & $\mathrm{Si}$ & 133 & 14,9 & 20 & 65 & \\
\hline \multirow[t]{3}{*}{ Consumo alcohol } & Normal & 558 & 62,4 & 11 & 35,5 & 0,000 \\
\hline & Alto riesgo & 263 & 29,4 & 10 & 32,3 & \\
\hline & Alcoholismo & 73 & 8,2 & 10 & 32,3 & \\
\hline \multirow[t]{3}{*}{ Consumo drogas } & Normal & 671 & 75,1 & 12 & 38,7 & 0,000 \\
\hline & Alto riesgo & 181 & 20,2 & 14 & 45,2 & \\
\hline & Dependencia drogas & 42 & 4,7 & 5 & 16,1 & \\
\hline
\end{tabular}

*Prueba de chi cuadrado

En cuanto a la fuerza de asociación de la cohesión familiar con las características demográficas, personales y de consumo de los adolescentes, se encontró que, respecto al sexo la probabilidad de buena cohesión familiar es dos veces en los hombres respecto a las mujeres (OR crudo=2,13; IC95\% 1,013$4,41)$. La cohesión familiar mayor en los adolescentes de colegios privados (OR crudo=3,38; IC95\% 1,29$8,88)$.

La frecuencia de satisfacción familiar en los adolescentes con cohesión familiar alta, encontrándose que fue 25 veces más probable que haya cohesión familiar en estos adolescentes satisfechos (OR crudo $=25,22$; IC95\% 10,61-59,94). Se encontró que es dos veces más probable la buena cohesión familiar en los adolescentes que manifestaron que en su hogar no hay violencia $(O R$ crudo=3,08; IC95\% 1,36-6,95).

Las habilidades sociales también se mostraron como factor asociado a la cohesión, observándose que la probabilidad de buena cohesión familiar es dos veces mayor en aquellos adolescentes con buenas habilidades sociales (OR crudo=2,97; IC95\% 1,41$6,23)$. En los adolescentes sin riesgo de depresión la probabilidad de cohesión familiar es 10 veces mayor que en aquellos con depresión (OR crudo=10,40; IC95\% 4,87-22,21); además esta probabilidad de buena cohesión familiar también fue siete veces mayor en aquellos que no tenían riesgo de alcoholismo, que en quienes fueron clasificados como alcohólicos $(O R=6,95$; IC95\% 2,85-16,93) y en quienes no tenían dependencia a drogas, que en quienes si eran dependientes de alguna sustancia $(O R$ crudo=6,66; IC95\% 2,24-19,78).

Al realizar el modelo ajustado, se identificó que las dos características fuertemente asociadas a la buena cohesión familiar, fueron la satisfacción con la familia y la ausencia de depresión; identificándose que los adolescentes satisfechos con su familia, tenían 12 veces la probabilidad de buena cohesión familiar que aquellos insatisfechos (OR ajustado=13,51; IC95\% $5,15-35,41)$, y en los que estaban libres de depresión la probabilidad de cohesión familiar fue cuatro veces mayor que en aquellos con riesgo de depresión (OR ajustado=5,72; IC95\% 2,18-15,03). (Tabla 2). 
Tabla 2. Características que mejor explican la cohesión familiar en adolescentes escolarizados

\begin{tabular}{|c|c|c|c|c|c|c|c|}
\hline \multirow{2}{*}{ Variable } & \multirow{2}{*}{ Categorías } & \multirow{2}{*}{ OR crudo } & \multicolumn{2}{|c|}{ IC $95 \%$} & \multirow{2}{*}{ OR ajustado } & \multicolumn{2}{|c|}{ IC 95\% } \\
\hline & & & Min & Max & & Min & Max \\
\hline \multirow[t]{2}{*}{ Grupo de edad } & $12-15$ años & 1,19 & 0,58 & 2,45 & 1,20 & 0,25 & 5,74 \\
\hline & 16-18 años & 1,00 & & & 1,00 & & \\
\hline \multirow[t]{2}{*}{ Sexo } & Masculino & 2,13 & 1,03 & 4,41 & 0,73 & 0,28 & 1,93 \\
\hline & Femenino & 1,00 & & & 1,00 & & \\
\hline \multirow[t]{2}{*}{ Tipo de colegio } & Público & 1,00 & & & 1,00 & & \\
\hline & Privado & 3,03 & 0,41 & 22,50 & 2,55 & 0,82 & 7,91 \\
\hline \multirow[t]{3}{*}{ Grado escolaridad } & Sexto-séptimo & 1,00 & & & 1,00 & & \\
\hline & Octavo-noveno & 2,69 & 1,01 & 7,18 & 1,39 & 0,43 & 4,48 \\
\hline & Décimo-undécimo & 1,12 & 0,49 & 2,56 & 0,82 & 0,15 & 4,53 \\
\hline \multirow{2}{*}{ Satisfacción con la familia } & Satisfecho & 25,22 & 10,61 & 59,94 & 13,51 & 5,15 & 35,41 \\
\hline & Insatisfecho & 1,00 & & & 1,00 & & \\
\hline \multirow[t]{2}{*}{ Violencia intrafamiliar } & Ausencia & 3,08 & 1,36 & 6,95 & 1,38 & 0,53 & 3,58 \\
\hline & Presencia & 1,00 & & & 1,00 & & \\
\hline \multirow[t]{2}{*}{ Déficit habilidades sociales } & No & 2,97 & 1,41 & 6,23 & 1,11 & 0,44 & 2,79 \\
\hline & $\mathrm{Si}$ & 1,00 & & & 1,00 & & \\
\hline \multirow{2}{*}{ Depresión } & No & 10,40 & 4,87 & 22,21 & 5,72 & 2,18 & 15,03 \\
\hline & $\mathrm{Si}$ & 1,00 & & & 1,00 & & \\
\hline \multirow[t]{3}{*}{ Consumo de alcohol } & Normal & 6,95 & 2,85 & 16,93 & 2,28 & 0,67 & 7,77 \\
\hline & Alto riesgo & 3,60 & 1,44 & 8,99 & 2,14 & 0,69 & 6,70 \\
\hline & Alcoholismo & 1,00 & & & 1,00 & & \\
\hline \multirow[t]{3}{*}{ Consumo de drogas } & Normal & 6,66 & 2,24 & 19,78 & 1,04 & 0,25 & 4,27 \\
\hline & Alto riesgo & 1,54 & 0,53 & 4,51 & 0,86 & 0,24 & 3,10 \\
\hline & Dependencia drogas & 1,00 & & & 1,00 & & \\
\hline
\end{tabular}

\section{Discusión}

La familia tiene un papel protagónico en el desarrollo psicosocial del adolescente, participando de manera activa en la formación de capacidades cognitivas, comportamentales, sociales, morales y culturales del adolescente, necesarias para la transición de la niñez a la adultez; éste es un proceso altamente variable entre los individuos, condicionado por diferentes circunstancias. Las relaciones familiares en este estudio fueron medidas a través del clima $o$ satisfacción familiar, expresado en la manera como los adolescentes perciben las relaciones entre la familia; así mismo, se evaluó la cohesión familiar, enfocada en la conexión familiar entre los miembros de la familia, que puede asemejarse al apoyo familiar(18).

Los participantes de esta investigación en su mayoría tenían entre 12 y 15 años y se encontraban en octavo y noveno grado escolar; se sabe que la edad trae consigo ciertas tareas propias del desarrollo, dentro de ellas la autonomía, la cual se logra al independizarse económica y emocionalmente, migrando su centro emocional de los padres hacia la pareja, situación característica de la adolescencia media, rango de edad en el que se encuentran la mayoría de los jóvenes encuestados ${ }^{(1)}$.

En la adolescencia una característica importante es la adaptabilidad a situaciones estresantes propias de las condiciones sociales y la capacidad de resiliencia a los acontecimientos vitales del ciclo familiar. En este sentido, se observó que a pesar de que, en algunas familias, según la percepción del adolescente, hubiera violencia, se observó una mayoría de participantes satisfechos con su familia y quienes manifestaron una buena cohesión familiar. Al respecto, de acuerdo con Zuñeda et al.,(6) a menor violencia intrafamiliar mayor cohesión y satisfacción con la familia.

La adolescencia media (12 a 14 años) es una etapa en la cual la satisfacción con la familia y la percepción de cohesión familiar puede ser mayor respecto a los adolescentes mayores(19). En este sentido, los hallazgos de la presente investigación mostraron que, en los adolescentes pertenecientes a instituciones públicas y privadas, esta satisfacción con las características propias de la familia fue mayor en la adolescencia media. La poca satisfacción con la familia en la adolescencia tardía (15 a 17 años) puede encontrar explicación en el hecho de que en este 
rango de edad los jóvenes dan mayor importancia el grupo de pares y a nuevas relaciones sociales.

A pesar de que algunos autores como Zdanowicz et $a l$. ,(20) indican que no hay diferencias en las percepciones de cohesión familiar respecto al género, en la presente investigación se observó una mayor percepción de adecuada cohesión en los hombres.

Una adecuada cohesión familiar mostró una estrecha relación con la satisfacción percibida por el adolescente respecto a su familia. Al respecto, autores como Verdugo et al.,(21) encontraron en un estudio realizado en el año 2014, que los jóvenes con mayor cohesión familiar presentaban mayor satisfacción con el clima familiar, lograron así una mejor adaptación social al medio, lo cual puede darse porque a mayor tiempo compartido en familia, mejor respuesta social del joven.

La cohesión familiar y en el mismo sentido la buena satisfacción de los adolescentes con sus familias repercute significativamente en los estados de salud mental y relaciones sociales de los adolescentes, sustentada esta idea en los hallazgos de la presente investigación, donde la buena cohesión fue factor clave para la ausencia de presencia de riesgo de depresión y de consumo de sustancias como alcohol y drogas. En este sentido, autores como Gómez(18),

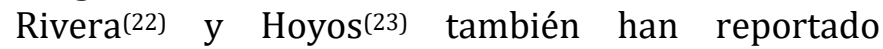
asociación entre la cohesión, comunicación y cercanía de los adolescentes con sus padres y la presencia o ausencia de depresión.

Las relaciones familiares distantes pueden favorecer conductas riesgosas en los adolescentes por la vulnerabilidad emocional que puede acarrear(5,24); un joven con adecuadas relaciones entre sus familiares y que cuenta con el apoyo permanente de éstos, tienen mejores estrategias para resolver los conflictos, además de un excelente desempeño en distintos ámbitos de la vida(25,26).

El contexto familiar en el que se desenvuelven los adolescentes puede influir negativa o positivamente en aspectos relacionados con la salud mental y en conductas de consumo de sustancias de los adolescentes, es en este espacio en el que se adquieren y fortalecen las distintas costumbres y se forjan aspectos relacionados con la personalidad y hábitos; es por esto, que pautas de interacción familiar, como baja cohesión, una mala comunicación, constantes conflictos, entre otras, repercuten de forma directa en la vida de los jóvenes $(27,28)$.

La adolescencia es una etapa de vulnerabilidad en la cual los jóvenes definen muchos aspectos biopsicosociales que los acompañaran durante las demás etapas de la vida; en esta, la familia juega un papel fundamental en la adquisición de conductas, por lo cual es importante el fomento de relaciones saludables dentro del núcleo familiar(29,30).

Durante la adolescencia las experiencias de vida que rodean al adolescente, marcan su autoestima y forjan su personalidad, por ello es fundamental la preparación de las familias para el afrontamiento de los conflictos y tensiones generados en medio de las relaciones internas, así como para fomento de un autoestima positiva en los adolescentes y el manejo de diversas situaciones de riesgo que pueden surgir en la vida, como lo es el consumo de sustancias psicoactivas que pueden desencadenar dependencias $\mathrm{y}$ adicciones( ${ }^{(31,32)}$.

\section{Conclusiones}

Si bien los jóvenes y adolescentes crecen en contextos en los cuales el acceso a drogas y alcohol puede llevarlos a un riesgo mayor de consumo y dependencia, en estos casos, una buena cohesión familiar es clave para prevenir comportamientos de riesgo en los adolescentes. En este sentido, el fortalecimiento de las relaciones familiares y el logro de altos niveles de cohesión familiar, puede potenciarse generando sentimientos de agrado y comodidad en el adolescente frente a su entorno familiar, así mismo, trabajando en el cuidado de la salud mental del joven.

Los hallazgos aportan a tomadores de decisión en salud pública, insumos para la prevención de comportamientos de riesgos en jóvenes y adolescentes, así como el desarrollo de futuras investigaciones que evalúen el efecto positivo de intervenciones colectivas en el fortalecimiento de las relaciones familiares.

Conflicto de intereses: Ninguno declarado por los autores.

\section{Referencias}

1. Gaete V. Desarrollo psicosocial del adolescente. Revista Chilena de Pediatría. 2015;86(6):436-43. DOI: 10.1016/j.rchipe.2015.07.005. 
2. Jaggers JW, Church WT, Tomek S, Hooper LM, Bolland KA, Bolland JM. Adolescent Development as a Determinant of Family Cohesion: A Longitudinal Analysis of Adolescents in the Mobile Youth Survey. Journal of Child and Family Studies. 2015;24(6):1625-37. DOI: 10.1007/s10826-014-9966-8.

3. Villarreal-Zegarra D, Paz-Jesús A. Family Cohesion, Adaptability and Composition in Adolescents from Callao, Peru. Propósitos y Representaciones [Internet]. 2017 [citado 5 de septiembre de 2020];5(2):21-64. Disponible en: http://revistas.usil.edu.pe/index.php/pyr/article/view/15 8

4. Martínez Pampliega A, Ugarte Elicegui I, Merino L, Herrero Fernández D. Conciliación familia-trabajo y sintomatología externalizante de los hijos e hijas: papel mediador del clima familiar. Rev iberoam psicol salud. 2019;10(1):27-36. DOI: 10.23923/j.rips.2018.02.023.

5. Buitrago Matamoros JF, Pulido Archila LY, Güichá-Duitama ÁM. Relación entre sintomatología depresiva y cohesión familiar en adolescentes de una institución educativa de Boyacá. PSICOGENTE [Internet]. 2017 [citado 5 de septiembre de 2020];20(38):296-307. Disponible en: http://revistas.unisimon.edu.co/index.php/psicogente/arti cle/view/2550

6. Zuñeda A, Llamazares A, Marañón D, Vázquez G. Características individuales y familiares de los adolescentes en violencia filio-parental: la agresividad física, la cohesión familiar $y$ el conflicto interparental como variables explicativas. RPPC [Internet]. 2016 [citado 20 de octubre de 2020];21(1):21-33. Disponible en: http://revistas.uned.es/index.php/RPPC/article/view/150 21

7. Matienzo-Manrique ME. Aporte del funcionamiento familiar en las conductas antisociales en estudiantes de secundaria. CASUS. 2020;5(1):8-17. DOI: 10.35626/casus.1.2020.233.

8. Segrin C, Kauer TB, Burke TJ. Indirect Effects of Family Cohesion on Emerging Adult Perfectionism Through Anxious Rearing and Social Expectations. J Child Fam Stud. 2019;28(8):2280-5. DOI: 10.1007/s10826-019-01444-2.

9. Ahookhosh P, Bahmani B, Asgari A, Hassanian Moghaddam H. Family Relationships and Suicide Ideation: The Mediating Roles of Anxiety, Hopelessness, and Depression in Adolescents. Int J High Risk Behav Addict [Internet]. 2016 [citado 20 de octubre de 2020];6(1):e31573. Disponible en: https://sites.kowsarpub.com/ijhrba/articles/13233.html

10. Aguirre-Guiza NC, Aldana-Pinzon OB, Bonilla-Ibáñez CP. Factores familiares de riesgo de consumo de sustancias psicoactivas en estudiantes de una institución de educación media técnica de Colombia. Rev salud pública. 2017;19(1):39. DOI: 10.15446/rsap.v19n1.41785.

11. Esteves Villanueva AR, Paredes Mamani RP, Calcina Condori CR, Yapuchura Saico CR. Habilidades Sociales en adolescentes y Funcionalidad Familiar. Comuni@cción. 2020;11(1):16-27. Disponible

en: http://www.scielo.org.pe/scielo.php?script=sci_abstract\&pi $\mathrm{d}=\mathrm{S} 2219-71682020000100016$

12. Torres de Galvis Y, Posada Villa J, Berbesi Fernández DY. Factores asociados al abuso y la dependencia de sustancias en adolescentes colombianos, con base en los resultados del WMH-CIDI. Revista Colombiana de Psiquiatría. 2010;39(Suppl 1):112-32. Disponible en: http://www.scielo.org.co/scielo.php?script=sci_arttext\&pid =S0034-74502010000500009

13. Torres de Galvis Y, Osorio Gómez JJ, López Bustamante PL. Salud mental del adolescente, Medellín 2006. Medellín: CES;
2007.

Disponible

en:

https://www.medellin.gov.co/irj/go/km/docs/wpccontent /Sites/Subportal\%20del\%20Ciudadano/Salud/Secciones/P ublicaciones/Documentos/2012/Investigaciones/Libro\%2 0Salud\%20Mental\%20del\%20Adolescente\%202006.pdf

14. Maya J, Torres de Galvis Y, Murrelle L. Consumo de sustancias psicoactivas y otros factores asociados con delincuencia juvenil. Medellín: Marín Vieco; 2000.

15. Cogollo Z, Diaz CE, Campo A. Exploración de la validez de constructo de la escala de Zung para depresión en adolescentes escolarizados. Colombia Médica. 2006;37(2):102-6. Disponible en: http://www.scielo.org.co/scielo.php?pid=S1657$95342006000200004 \&$ script $=$ sci_abstract\&tlng $=e s$

16. Gismero E. EHS, Escala de habilidades sociales. TEA Ediciones; 2010.

17. Vinaccia S, Quiceno J, Fernández $H$, Naranjo $M$. Autoesquemas y habilidades sociales en adolescentes con diagnóstico de labio y paladar hendido. Pensamiento Psicológico. 2008;4(10):123-35. Disponible en: https://www.redalyc.org/articulo.oa?id=80111670008

18. Gómez-Restrepo C, de Santacruz C, Rodríguez MN, Rodríguez V, Tamayo Martínez N, Matallana D, et al. Encuesta Nacional de Salud Mental Colombia 2015. Protocolo del estudio. Revista Colombiana de Psiquiatría. 2016;45(1):2-8. Disponible https://www.redalyc.org/articulo.oa?id=80650839002

19. Morón Marchena JA, Pérez-Pérez I, Pedrero García E. Educación para la salud y prevención de riesgos psicosociales en adolescentes y jóvenes. Madrid: Narcea; 2017;28(4):1343-5. DOI: 10.5209/RCED.56016

20. Zdanowicz N, Pascal J, Reynaert C. ¿Juega la familia un papel clave en la salud durante la adolescencia? The European journal of psychiatry [Internet]. 2004;18(4):215-24. Disponible en: http://scielo.isciii.es/scielo.php?script=sci_arttext\&pid=S15 79-699X2004000400003

21. Verdugo Lucero JC, Arguelles Barajas J, Guzmán Muñiz J, Márquez González C, Montes Delgado R, Uribe Alvarado I. Influence of family environment in the adolescent's social adaptation process. Psicología desde el Caribe. 2014;31(2):207-22. Disponible en: https://www.redalyc.org/articulo.oa?id=21331836002

22. Rivera CR, Arias-Gallegos WL, Cahuana-Cuentas M. Perfil familiar de adolescentes con sintomatología depresiva en la ciudad de Arequipa, Perú. Rev chil neuro-psiquiatr. 2018;56(2):117-26. DOI: $\quad 10.4067 / \mathrm{s} 0717$ 92272018000200117.

23. Hoyos Zuluaga E, Lemos Hoyos M, Torres de Galvis Y. Risk factors and protective adolescent depression in the city of Medellín. Int j psychol res. 2012;5(1):109-21. DOI: 10.21500/20112084.767.

24. Poole LA, Lewis AJ, Toumbourou JW, Knight T, Bertino MD, Pryor R. A Multi-Family Group Intervention for Adolescent Depression: The BEST MOOD Program. Fam Proc. 2017;56(2):317-30. DOI: 10.1111/famp.12218.

25. Ossa Hernández A, Sánchez Ochoa A, Mejía Vasco CE. Prevalencia de depresión y sus factores asociados en adolescentes entre 13 y 17 años en Colombia año 2003. CES Salud Pública. 2016;7(1):37-48. DOI: 10.21615/cessp.7.1.4.

26. Andrade-Sánchez A, Galindo-Villardon M, Salazar C. Caracterización de los hábitos de vida, percepción y apoyo social en estudiantes preuniversitarios mediante el algoritmo taid. Invest Oper. 2019;40(2):192-200. Disponible 
en:

http://www.invoperacional.uh.cu/index.php/Inv0p/article /view/661

27. Musitu G, Jiménez T, Murgui S. Funcionamiento familiar, autoestima y consumo de sustancias en adolescentes: un modelo de mediación. Salud Pública México. 2007;49(1):310. Disponible en: http://www.scielo.org.mx/scielo.php?script=sci_arttext\&pi $\mathrm{d}=\mathrm{S} 0036-36342007000100002$

28. González Quijada E, González Portillo M, Marín Marín M. La familia y la escuela en la construcción de valores. Un enfoque cualitativo. Diálogos educativos. 2009;9(17):58-73. Disponible

en: https://dialnet.unirioja.es/servlet/articulo?codigo=303906 8

29. Telumbre-Terrero JY, López-Cisneros MA, Castillo-Arcos L del C, Sánchez Becerra A, Sánchez-Domínguez JP. Family history and alcohol consumption in adolescents. Revista Salud Uninorte. 2019;35(1):55-66. Disponible en: https://rcientificas.uninorte.edu.co/index.php/salud/articl e/view/8623
30. Morales Gonzáles F, Cabrera Jiménez M, Pérez Barajas CJ, Amaro Franco ML. El consumo de alcohol en adolescentes de una secundaria de Ciudad Nezahualcóyotl. Frecuencia y características sociales. Vertientes Revista especializada en salud. 2015;18(1):8-15. Disponible en: http://www.revistas.unam.mx/index.php/vertientes/articl e/view/51727/46168

31. Díaz Falcón D, Fuentes Suárez I, Senra Pérez $\mathrm{N}$ de la C. Adolescencia y autoestima: su desarrollo desde las instituciones educativas. Conrado. 2018;14(64):98-103. Disponible en: http://scielo.sld.cu/scielo.php?script=sci_arttext\&pid=S199 0-86442018000400098

32. Castaño Castrillón JJ, Páez Cala ML. Funcionalidad familiar y tendencias adictivas a internet y a sustancias psicoactivas en estudiantes universitarios. Psicología desde el Caribe. 2020;36(2):177-206. Disponible en: https://rcientificas.uninorte.edu.co/index.php/psicologia/a rticle/view/10090 